\title{
COMPUTER ANIMATION WITH CINEMA
}

\author{
David R. Kalasky \\ Deborah A. Davis \\ Systems Modeling Corporation \\ The Park Building \\ 504 Beaver Street \\ Sewickley, PA 15143
}

\begin{abstract}
CINEMA is a general-purpose animation system designed to animate models developed using the SIMAN simulation language. The CINEMA package consists of a module used to draw the graphical images used in the animation and a module to execute a SIMAN model and associated animation.

Animation typically centers around the presentation of final modeling results. However, the simplicity of developing CINEMA animations allows users to exploit animation in more phases of a simulation project. By using CINEMA, animation benefits can be found throughout the entire simulation project from initial model building through the presentation of the final simulation results.

CINEMA IV complements the advanced capabilities found in SIMAN. While any process can be modeled using SIMAN/CINEMA, the software has exceptional ease and accuracy in modeling material handling functions such as accumulating conveyors and AGV systems. CINEMA provides real-time graphical outputs for SIMAN statistical routines such as dynamic plots and histograms.

CINEMA is available on a wide variety of platforms and operating systems. These include microcomputers under DOS and OS/2, as well as Apollo, IBM, Sun, and VAX workstations under the AIX Windows, UNIX, and VMS operating systems.
\end{abstract}

\section{INTRODUCTION}

In recent years animation has become a requirement of the simulation process. One of the reasons for this requirement is that numeric summary statistics do not necessarily convey information about the dynamic interactions of components of a system. Although summary statistics are a crucial part of evaluating the performance of a simulated system, it is only through animation that the analyst can easily identify the system status under which, for example, bottlenecks occur.
Using the general purpose simulation language SIMAN (Pegden, 1990), in conjunction with the CINEMA animation system, offers a means of analyzing both the system performance and the dynamics during the simulation of any discrete-event system.

Benefits of animation are not restricted to bottleneck analysis only. Johnson and Poorte (1988) identified four simulation modeling phases where the modeler may benefit from using animation. They are:

1. Model Building and Verification

2. Model Validation

3. Bottleneck Analysis

4. Communication and Presentation

\subsection{Model Building and Verification}

Animation provides a tool to visually inspect that a model acts and reacts as intended. By viewing the animation, logical errors are easily identified. This added view of the model in action reduces the likelihood of inaccurate modeling. Therefore, invalid conclusions, incorrect decisions, and large failure costs may be avoided.

\subsection{Model Validation}

Validation requires thorough knowledge and understanding of the intricacies of the system under consideration. The analyst may have the required awareness but by using simulation only, one can never be totally sure that the model is a good representation of the real system. Modeled projects require input and review by several different people. The reviewers typically have varying degrees of modeling and process expertise. Animations provide a common data base of communication concerning the systems being evaluated.

Animation provides the analyst with a powerful tool to ensure that the model is indeed a sufficiently adequate representation of the real system. For example, the impact that a simplification may have on the model is readily seen in a running animation. Assumptions that have a profound effect on the 
performance of a model may go unnoticed without the use of animation, which, again, could lead to inaccurate results and incorrect conclusions. As a model validation tool, animation allows the modeler to make reasonable simplifications and other model assumptions.

\subsection{Bottleneck Analysis}

As an analysis tool animation can play a major role in bottleneck analysis. Using animation, the modeler has the ability to observe interactions of several simultaneous and interrelated events, thus providing information unavailable in aggregate statistical performance measures. Bottleneck situations are usually simple to locate with animation. Also, the important pre-conditions under which these and other unfavorable situations occur can be identified. By observing an animation, these factors become apparent and they may also help visualize candidate improvements.

\subsection{Communication and Presentation}

The ultimate objective of any simulation project is to provide credible information to the decision maker. Credibility of a project is strongly associated with the ability of the modeler to effectively communicate throughout a simulation project. Animation gives the analyst a very powerful vehicle to provide the nonsimulationist with insight into a complex model. Likewise, a "process expert" can visualize and appreciate the simulation methodology.

It should be stressed that animation cannot replace standard statistical analysis techniques. However, the above discussion illustrates that with animation the modeler has access to a powerful analysis tool. As such, animation has the ability to effectively change and enhance virtually all phases of the modeling process.

\section{DESIGN PHILOSOPHY OF CINEMA}

Designing and building realistic simulation models is a complicated and often time-consuming process. CINEMA was designed for the user to exploit animation throughout a simulation project from initial model building through the presentation of the final results. Certain features of the architecture of the CINEMA animation package allows for this versatility.

Three main design objectives for CINEMA were:

1) Simplicity

2) Flexibility

3) Effectiveness

CINEMA's simplicity of use permits easy mastery of the task of building an animation; its flexibility allows graphical animation of any system at any level of detail; and its effectiveness greatly assists in communication throughout a simulation study.

\subsection{Simplicity}

A common objective for software architects is that their products be easy to use. One key element in the design of CINEMA, is its ease of use; no special training is required to readily construct impressive animations. A mouse-controlled user-interface where users select items from pop-down menus is the way in which animation layouts are developed. With CINEMA, no programming is required to build an animation. The user can fully concentrate on developing useful, realistic models and the data to support the analysis.

\subsection{Flexibility}

The CINEMA animation package is intimately tied to the SIMAN simulation language. SIMAN is a general purpose language and has been used in a wide variety of environments, such as manufacturing, communication, health care, and defense. CINEMA is used in the same environments, therefore the same flexibility is incorporated in the animation.

Johnson and Poorte (1988) have proposed a hierarchical approach to computer animation in simulation modeling. Under their approach, animation is used during all phases of a modeling project. Animations evolve from simple modeling tools, to interactive analysis tools, until the final presentation of the results as a powerful communication vehicle. The associated animations are of increasing visual accuracy and detail. The authors support CINEMA as unique in providing the flexibility necessary for a multi-level approach to computer animation.

\subsection{Effectiveness}

Effectiveness of an animation system is measured by how valuable the graphical images are during the different phases of a simulation project. CINEMA's design assures effective use of animation. It can play the role of a verification and validation tool in the early phases of a project. In the next phase CINEMA becomes a powerful analysis tool in which bottlenecks can be detected and many "what- if" questions can be explored interactively. In the final phase of a project, impressive animations can be constructed with CINEMA and it becomes a communication tool highlighting key features for targeted audiences. 


\section{BUILDING A CINEMA ANIMATION}

The user interacts with CINEMA by using a mousecontrolled graphics cursor to pick items from pop-down menus as shown in Figure 1. Context sensitive help is available online simply by pointing to an item and clicking the right mouse button. The left mouse button activates the currently highlighted function.

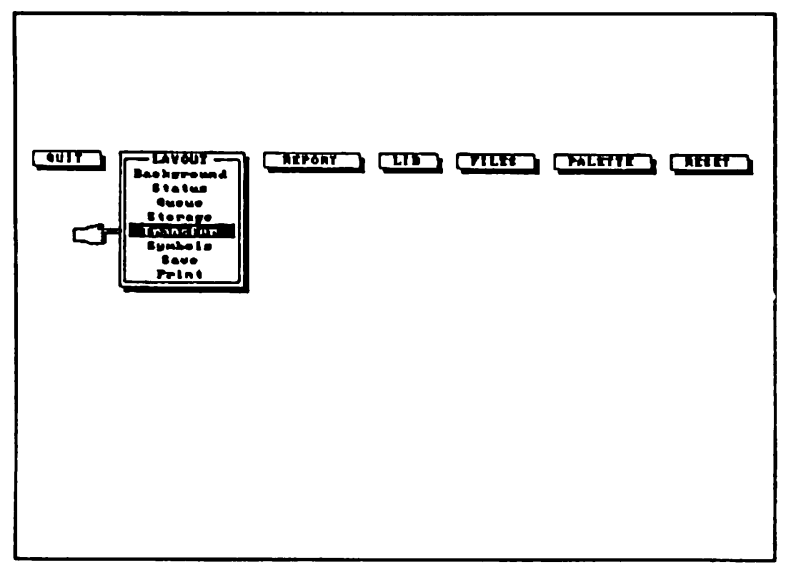

Figure 1: The CINEMA Interface

The CINEMA layout is a graphical representation of the system being simulated. The layout consists of graphical objects grouped into two categories: static and dynamic. The static component of the layout does not change during the execution of the simulation, while the dynamic component consists of graphical images which do change color, location, and shape.

\subsection{The Static Component}

The static component of a layout represents the physical environment in which the simulated system exists. It includes anything that helps to visually recognize the scene, but does not change during model execution. The static component (called background in CINEMA) can be created by using the drawing facilities provided in the CINEMA program. Under the draw menu drawing functions are available for line, polyline, box, circle, bar (a filled box), arc, and freehand sketch. Drawing options can be modified by selecting a different color, line style, and/or line thickness for each drawing function. Text can be added to the background at any location and in any orientation. Text options include any combination of six different sizes, with six different fonts, and 16 active colors. Explode allows the user to zoom in for fine detailing of small portions of the drawing, while Cut Area provides a means of copying or moving rectangular regions of the background or saving regions to files for use in other CINEMA animations.

An alternate method for generating a background is to start with a DXF-formatted file generated by a computer-aided design (CAD) packages such as AutoCAD, PC-CAD, CADVANCE, etc. Many of these packages have sophisticated drawing functions, especially for three-dimensional images, allowing the user to develop geometrically accurate and more complex backgrounds. DXF-formatted files generated on any hardware platform can be input into CINEMA. An example of a layout generated via this CINEMA utility is illustrated in Figure 3.

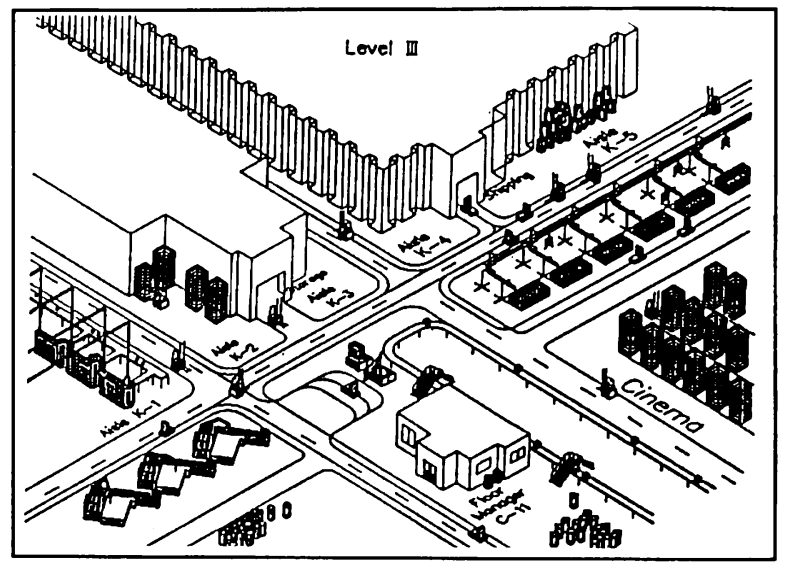

Figure 2: CINEMA Layout Imported From a CAD Drawing.

By using CAD drawings and CINEMA the user obtains the best of both worlds. For example, AutoCAD has powerful 3-D drawing commands, whereas CINEMA contains functions that cannot be performed by CAD packages, such as area fills and pixel-based editing. Impressive displays have been generated by using AutoCAD to develop the "technical part" of the layout followed by CINEMA to fine tune the "aesthetic part" of the layout.

\subsection{The Dynamic Component}

The dynamic component of the layout consists of graphical images which change shape, color, size, or location in response to a status change in the SIMAN model. The dynamic objects in a CINEMA layout are directly tied to SIMAN modeling constructs. As the state of the constructs change during execution, the associated dynamic objects will change to reflect this change of the SIMAN construct. A large number of dynamic objects are available; each one is discussed in some detail below. 
Entities - Entities represent the items that are being processed or flow through the system. Entity symbols are created by drawing the icon on a blown-up grid ('fat bits'), using the mouse-controlled cursor. As the symbol is created on this grid, it is simultaneously displayed in actual size in the upper left corner of the screen, as shown in Figure 3.

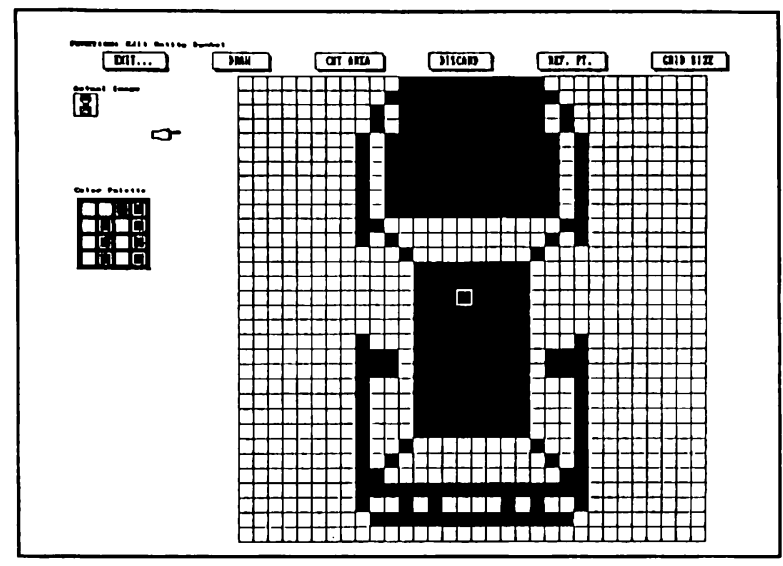

Figure 3: Creating an Entity Symbol

In CINEMA, a specific symbol from an entity library is associated with a unique attribute number. When the animation attribute changes values in the SIMAN model, the corresponding entity symbol changes in the animation. Consider, for example, a simulation of an automotive assembly plant. The entity arriving to an assembly station might have a value that corresponds to a symbol of a car body without doors (Figure 3). After leaving the workstation, the symbol could be changed to a car body with doors (Figure 4) simply by reassigning the symbol number in the SIMAN model.

Resources - Resources are used in SIMAN to model limited items in a system, such as machines and workers. Like entity symbols, resource symbols are created by drawing icons on the enlarged grid with the mouse. Resources dynamically change in a system between idle, busy, inactive, or preempted. Resource status changes within a SIMAN model are displayed in a CINEMA animation by using resource symbols that represent the resource in each of the possible states.

Queues - A queue can be added to the layout at any location, and in any length and orientation representing a SIMAN QUEUE block. These entities might represent workpieces awaiting the availability of a machine, a set-up operator, cars at a door assembly station, etc. When an entity enters a queue in the SIMAN model, the entity symbol is displayed along the corresponding queue symbol at the proper location relative to the other members of the queue. When an entity exits the queue in the model, its associated symbol is removed and all following symbols are moved forward one position. Sorting and prioritization can occur within queues and viewed via CINEMA.

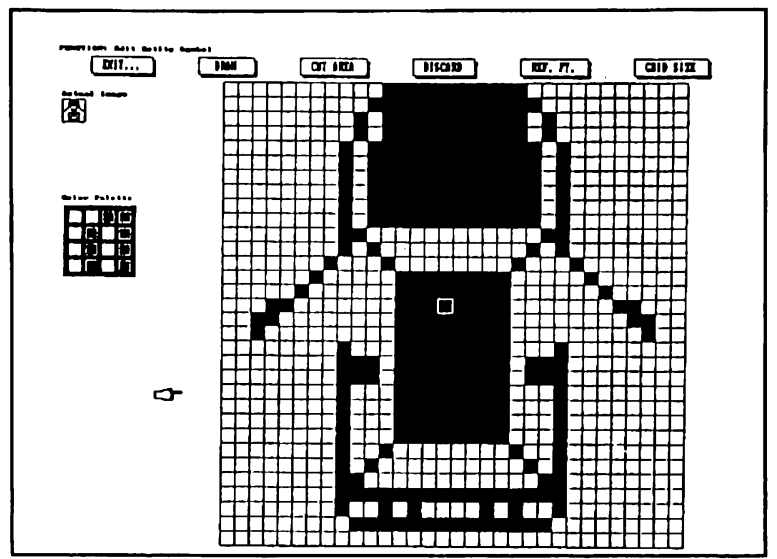

Figure 4: Subsequent Entity Symbol

Transfers - CINEMA's transfer menu is used to define the paths of travel for ROUTES, CONVEYORS, and TRANSPORTERS. STATION symbols are used to designate start and end points for the movement of entities. One method for modeling the movement of entities between STATIONS is to use the ROUTE block. When an entity reaches a ROUTE block in the SIMAN model, its entity symbol is continually redisplayed at new points along a predefined CINEMA route path to produce the effect of movement.

Movement that involves SIMAN material handling constructs is designated in much the same way as the routes. If entities are transferred by a CONVEYOR, the user will digitize paths called segments, and if the entities are moved by TRANSPORTERS, the paths will be called distances. All route, segment and distance paths are transparent during model execution, yet the entity symbols will trace these paths as they move.

Variables - While a simulation is executing, SIMAN automatically maintains the value of status variables which define the system state. Examples of these status variables are: simulation time, queue values, throughput, resource utilization, etc. Any SIMAN status variable can be incorporated into an animation layout using one of five dynamic features in CINEMA.

1) Digital displays can be added to a layout using a feature called dynamic variables. Users can tailor the format for display, range of values, size, and color using pull-down menus.

2) A second way to display status variables is with an analog representation of a variable's value. Three 
different level shapes are included in CINEMA: a box, a circle, and a dial. During execution of an animation, box or circle levels fill and empty in response to changes in the value of the associated status variable. The dial is a circular level with a sweep hand that rotates either clockwise or counter-clockwise. Dials are typically used to represent simulated time with a clock.

3) A feature called global symbols represents a third way to display the value of a status variable. Like entity and resource symbols, global symbols are drawn on the enlarged grid. For example, a symbol saying "STARVED" could be displayed when the number in a queue for a machine is zero, and a second symbol saying "BLOCKED" could be displayed when the queue is full to capacity.

4) Dynamic colors represents a fourth way to display the value of a status variable. While global symbols indicate specific conditions, dynamic colors can be assigned to indicate gradual state changes.

5) Plots and Histograms can also be displayed on the animation to show status variables changing over time.

\section{RUNNING AN ANIMATION}

Discussion so far has focused on the capabilities of the layout generation module of CINEMA. The execution of the SIMAN model in the CINEMA package has the ability to update the dynamic component of the layout interactively during execution as well as control the speed of execution. Since CINEMA is a real-time animation system, as opposed to a post-processed system, the menu includes facilities to temporarily stop a run and use the SIMAN interactive features. The interactive mode allows a user to change variables, look at the entities in a particular queue, look at status variables not displayed on the animation, and monitor practically any variable in the system. A user can turn on the SIMAN trace function so that the animation can be viewed simultaneously with the model execution statements. This feature is very useful for model verification and validation. A stepping facility halts the model execution after each event is processed. Execution is resumed when the user presses the space bar and continues until the time the next event is processed.

The user may associate any number of layouts with a single SIMAN model. These layouts are identified in files and may be recalled from the menus or directly from the keyboard. Figure 5 shows three layouts for a single SIMAN model. The first shows the entire facility while the other two layouts show detail of two different work centers. Another CINEMA feature is to save a snapshot. This saves a picture of the layout at a specific instant in simulated time as well as saving the value of all the system variables. The snapshot may be recalled at a later time so that the simulation can progress from the time that the snapshot was saved. This feature is particularly useful in demonstrating and presenting critical situations and comparing variations of the system at the same moment in time.

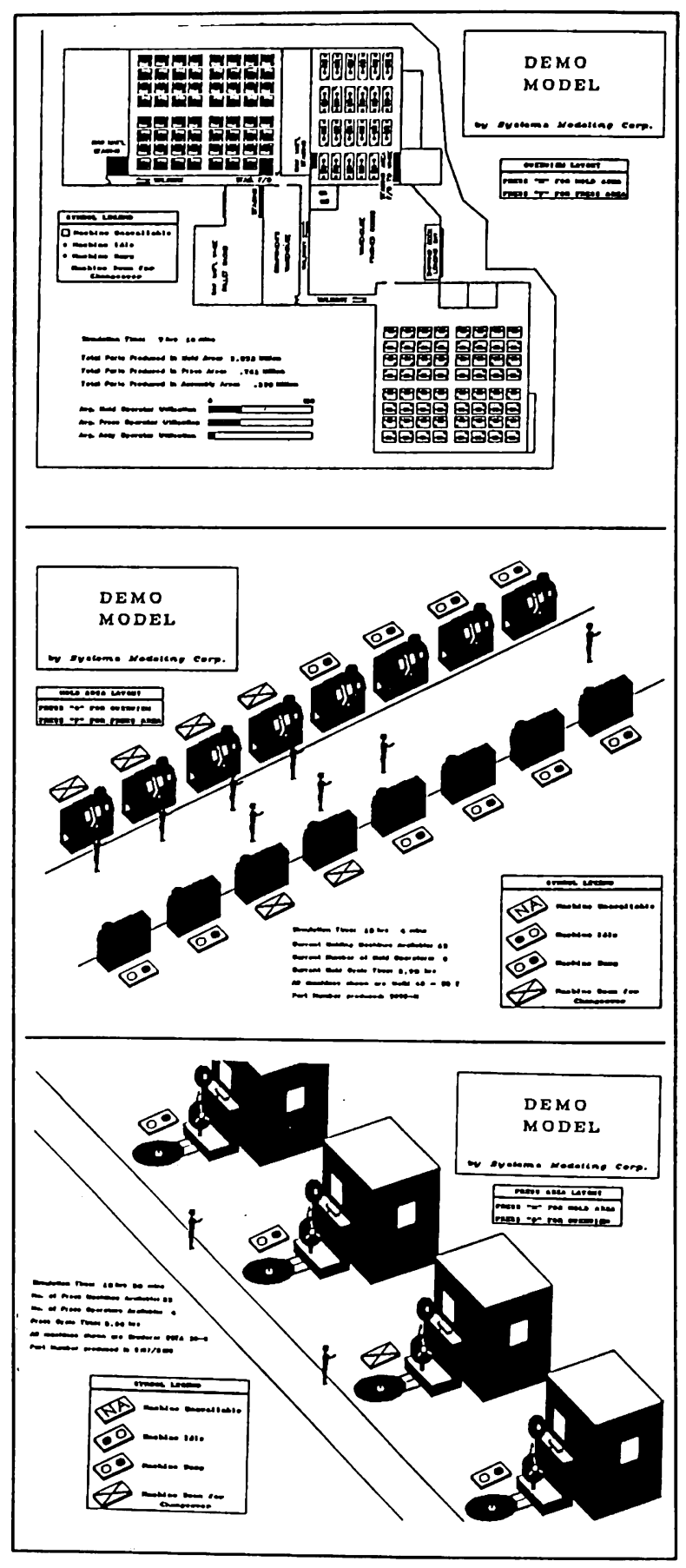

Figure 5: Multi-Layout Animated Model 


\section{MULTI-PLATFORM SUPPORT}

Since the initial release of CINEMA in 1986, many new platforms have been added. The primary platform has been the IBM PC-AT (or compatible) and the DOS operating system. There are currently three graphic cards available for the PC-AT/DOS configuration, namely the EGA card (or VGA card in EGA mode), a high-resolution card (HGA) purchased through Systems Modeling, or the IBM 8514/A card. The EGA has a pixel resolution of $640 \times 350$ while the high-resolution cards (HGA and 8514) have a pixel resolution of $1024 \times 768$. OS $/ 2$ allows simulation of systems virtually unlimited in size.

CINEMA is available on the Apollo DN3000 series workstation, Digital Equipment Corporation (DEC) VAXstation series of workstations (including MicroVAX workstations with the GPX upgrade), IBM RS-6000 workstations, and Sun Microsystems Sparc series workstations.

\section{SUMMARY}

CINEMA is a general purpose animation system which allows for the easy animation of any SIMAN simulation model. Because CINEMA utilizes user constructed icons, any type of system ranging from manufacturing, to distribution, to health care, transportation, and communications may be animated.

Three main design objectives of CINEMA are: (1) simplicity, (2) flexibility, and (3) effectiveness. The mouse-oriented, menu-driven user interface allows for the rapid development of animations without the need for programming. CINEMA animations aid the analyst in the process of building and verifying SIMAN models. Other benefits of CINEMA can be found during model validation and analysis phases, as well as in the presentation of the final results.

\section{ACKNOWLEDGMENTS}

This tutorial was adapted from the "Computer Animation with Cinema" tutorial prepared by J.P. Poorte and D.A. Davis for the Proceedings of the 1990 Winter Simulation Conference.

\section{REFERENCES}

Johnson, M. E. and J. P. Poorte (1988), "A Hierarchical Approach to Computer Animation in Simulation Modeling. Simulation 50, 1, 30-36.

Kasales, C.J. and D.T. Sturrock (1991), "Introduction to SIMAN," in Proceedings of the 1991 Winter Simulation Conference, B. L. Nelson, W. D. Kelton, and G. M. Clark, Eds. IEEE, Piscataway, NJ.
Pegden, C.D. R.E. Shannon, and R.P. Sadowski (1990), Introduction to Simulation Using SIMAN, McGraw-Hill, New York, NY.

Poorte, J.P. and D.A. Davis (1990), "Computer Animation With Cinema," In Proceedings of the 1990 Winter Simulation Conference, O. Balci, R.P. Sadowski, and R.E. Nance, Eds. IEEE, Piscataway, NJ, 123-127.

\section{AUTHOR BIOGRAPHIES}

DAVID R. KALASKY is the Product Services Manager for Systems Modeling Corporation. Prior to joining Systems Modeling, Mr. Kalasky was Manager of Simulation for Westinghouse at their Corporate Productivity and Quality Center. Mr. Kalasky holds a B.S. degree in Industrial Engineering from the University of Kentucky and an M.S. degree in Operations Research from the Pennsylvania State University. His experience includes work in engineering and the management of production and quality as well as simulation consulting. $\mathrm{He}$ is a registered Professional Engineer, an active member of IIE and SCS, CPIM and instructor within APICS, invited author and speaker for SME, and was Business Chairman for the the 1990 Winter Simulation Conference.

DEBORAH A. DAVIS is the Vice President of Software Development with Systems Modeling Corportation (SM). Since joining SM in 1984, she has worked on development of SIMAN, CINEMA, and other SMC products. Miss Davis received B.S. and M.S. degrees in Industrial Engineering and Operations Research from the Pennsylvania State University. Her current interests include simulation language and user interface design, new applications of simulation technology, and object oriented methodologies. She is currently the Business Chair for the 1991 WSC, was the Exhibits Chair for the 1990 WSC and will be the General Chair of the 1994 WSC. She is a member of SCS, IIE, Tau Beta Pi, and Alpha Pi Mu. 